\title{
Efeito do Diterpeno Manool sobre a Pressão Arterial e Reatividade Vascular em Ratos Normotensos e Hipertensos
}

\author{
Effect of Diterpene Manool on the Arterial Blood Pressure and Vascular Reactivity in Normotensive and \\ Hypertensive Rats
}

Ariadne Santana e Neves Monteiro, ${ }^{1}$ Debora Ribeiro Campos, ${ }^{1}$ Agnes Afrodite Sumarelli Albuquerque, ${ }^{1}$ Paulo Roberto Barbosa Evora, ${ }^{1 \oplus}$ Luciana Garros Ferreira, ${ }^{1}$ Andrea Carla Celotto ${ }^{1}$

Universidade de São Paulo Faculdade de Medicina de Ribeirão Preto, ${ }^{1}$ Ribeirão Preto, SP - Brasil

\section{Resumo}

Fundamento: Diversos estudos têm mostrado que as classes de diterpenos exercem efeito significativo no sistema cardiovascular. Os diterpenos, em particular, estão entre os principais compostos associados às propriedades cardiovasculares, como a propriedade vasorrelaxante, inotrópica, diurética e a atividade hipotensora. Embora o mecanismo de vasorrelaxamento do manool seja visível, seu efeito sobre a pressão arterial (PA) ainda é desconhecido.

Objetivo: Avaliar o efeito hipotensor in vivo do manool e verificar o efeito de vasorrelaxamento ex vivo em anéis aórticos de ratos.

Métodos: Os animais foram divididos aleatoriamente em dois grupos: normotensos e hipertensos. $\mathrm{O}$ grupo normotenso foi submetido à cirurgia sham e adotou-se o modelo 2R1C para o grupo hipertenso. Realizou-se monitoramento invasivo da PA para testes com manool em diferentes doses (10, 20 e $40 \mathrm{mg} / \mathrm{kg}$ ). Foram obtidas curvas de concentração-resposta para o manool nos anéis aórticos, com endotélio pré-contraído com fenilefrina (Phe) após incubação com N $\omega$-nitroL-arginina metil éster (L-NAME) ou oxadiazolo[4,3-a]quinoxalina-1-ona (ODQ). Os níveis plasmáticos de óxido nítrico (NOx) foram medidos por ensaio de quimioluminescência.

Resultados: Após a administração de manool, a PA se reduziu nos grupos normotenso e hipertenso, e esse efeito foi inibido pelo L-NAME em animais hipertensos apenas na dose de $10 \mathrm{mg} / \mathrm{kg}$. O manool ex vivo promoveu vasorrelaxamento, inibido pela incubação de L-NAME e ODQ ou remoção do endotélio. Os níveis plasmáticos de NOx aumentaram no grupo hipertenso após a administração de manool. $\mathrm{O}$ manool induz o relaxamento vascular dependente do endotélio na aorta de ratos, mediado pela via de sinalização NO/cGMP e redução da PA, e também pelo aumento plasmático de NOx. Esses efeitos combinados podem estar envolvidos na modulação da resistência periférica, contribuindo para o efeito anti-hipertensivo do diterpeno.

Conclusão: Esses efeitos em conjunto podem estar envolvidos na modulação da resistência periférica, contribuindo para o efeito anti-hipertensivo do diterpeno. (Arq Bras Cardiol. 2020; 115(4):669-677)

Palavras-chave: Doenças Cardiovasculares; Hipertensão; Diterpeno; Manool; Reatividade; Plantas Medicinais; Óxido Nítrico; Ratos.

\begin{abstract}
Background: Many studies have shown that the diterpenoid classes exert a significant effect on the cardiovascular system. Diterpenes, in particular, are among the main compound links to cardiovascular properties such as vasorelaxant, inotropic, diuretic and hypotensive activity. While the manool vasorelaxation mechanism is visible, its effect on blood pressure (BP) is still unknown.

Objective: To evaluate the in vivo hypotensive effect of manool and check the ex vivo vasorelaxation effect in rat aortic rings.

Methods: The animals were divided randomly into two groups: normotensive and hypertensive. The normotensive group was sham-operated, and the 2K1C model was adopted for the hypertensive group. Invasive BP monitoring was performed for manool tests at different doses $(10,20$ and $40 \mathrm{mg} / \mathrm{kg})$. Concentration-response curves for manool were obtained in the aorta rings, with endothelium, pre-contracted with phenylephrine (Phe) after incubation with N⿴-nitro-L-arginine methyl ester(L-NAME) or oxadiazole [4,3-a]quinoxalin-1-one (ODQ). Nitric oxide (NOx) plasma levels were measured by chemiluminescence assay.
\end{abstract}

Correspondência: Paulo Roberto Barbosa Evora •

Universidade de São Paulo Faculdade de Medicina de Ribeirão Preto - Cirurgia e Anatomia - Campus da USP. CEP 14040-900, Ribeirão Preto, SP - Brasil E-mail: prbevora@gmail.com

Artigo recebido em 27/03/2019, revisado em 27/08/2019, aceito em 23/10/2019

DOI: https://doi.org/10.36660/abc.20190198 
Results: After manool administration, BP was reduced in normotensive and hypertensive groups, and this effect was inhibited by L-NAME in hypertensive animals only in $10 \mathrm{mg} / \mathrm{kg}$ dose. Ex vivo manool promoted vasorelaxation, which was inhibited by L-NAME and ODQ incubation or endothelium removal. NOx plasma levels increased in the hypertensive group after manool administration. Manool elicits endotheliumdependent vascular relaxation in rat aorta mediated by the NO/CGMP signaling pathway and BP reduction, also by NOx plasma increase. These combined effects could be involved in modulating peripheral resistance, contributing to the antihypertensive effect of diterpene.

Conclusion: These effects together could be involved in modulating peripheral resistance, contributing to the antihypertensive effect of diterpene. (Arq Bras Cardiol. 2020; 115(4):669-677)

Keywords: Cardiovascular Diseases; Hypertension; Diterpenes; Manool; Reactivity; Nitric Oxide; Rats.

Full texts in English - http://www.arquivosonline.com.br

\section{Introdução}

Os diterpenos são uma ampla classe de metabólitos químicos, amplamente distribuídos no reino vegetal, com mais de 12.000 compostos conhecidos. ${ }^{1,2}$ Eles podem ser divididos em dois tipos: diterpenos de metabolismo especializado (secundário) e diterpenos de metabolismo geral (primário). Os diterpenos secundários podem ter funções nas interações ecológicas das plantas com outros organismos e benefícios em fármacos, perfumes, resinas e outros bioprodutos industriais com grande relevância econômica. ${ }^{1,2}$ Diversos metabólitos secundários, como terpenos, ácidos fenólicos, polifenóis, flavonoides e antocianinas, foram relatados em espécies de sálvia. Essas espécies são vistas como excelentes fontes de diterpenos. ${ }^{3}$ De acordo com os achados quimiotaxonômicos, o manool foi relatado anteriormente nas seguintes espécies de sálvia: S. sclarea, S. pubescens, S. lavandulifolia, S. hypoleuca, S. miltiorrhizae. Também está presente em outras espécies, como na Pinuscaribaea (Pinaceae), Lourteigiastoechadifolia (Asteraceae) e Halocarpusbiformis (Podocarpaceae). No entanto, o manool é o principal diterpeno das várias espécies de sálvia, sendo encontrado em maior concentração na Salvia officinalis. ${ }^{4}$

A biossíntese das unidades estruturais de isopreno de uma ampla variedade de terpenos, incluindo os diterpenos, ocorre pela via da desoxilulose. Essa via aumenta e evolui para dois produtos distintos: isopentenildifosfato (IPP) e dimetilalildifosfato (DMAPP). Mais especificamente, o manool, cuja composição química é $\mathrm{C}_{20} \mathrm{H}_{34} \mathrm{O}$, é um diperteno do tipo labdano bicíclico. Sua estrutura se baseia em um esqueleto carbonado do tipo $2 \mathrm{E}, 6 \mathrm{E}$, 10E-geranilgeranilpirofosfato (GGPP)..$^{5-7}$

A descoberta de novas substâncias com atividade antihipertensiva, baixo custo e poucos efeitos adversos é ainda um aspecto desejável e de importância para a utilização clínica. ${ }^{8}$ Porém, várias dificuldades são encontradas para esse fim, como a escolha do modelo experimental, obtenção de extratos padronizados e a dificuldade de obtenção, isolamento e identificação das substâncias ativas. ${ }^{9,10}$ A opção de conduzir pesquisas, a partir da indicação de plantas utilizadas pelas comunidades, encurta o percurso de desenvolvimento de um novo fármaco, pois os pesquisadores dispõem, antes mesmo de se iniciarem estudos científicos, de uma indicação de qual atividade biológica esta droga poderia apresentar. ${ }^{11,12}$

Os diterpenos, em particular, estão entre os principais compostos com ligação às propriedades cardiovasculares, tais como vasorrelaxante, inotrópica, diurética e hipotensiva. A ação vascular exercida por esses compostos parece envolver múltiplos mecanismos, como endotélio dependente e endotélio independente, aumento de prostaciclina e bloqueio de canais de cálcio dependentes de voltagem. ${ }^{13-17}$

Conforme descrito anteriormente na revisão da literatura, o manool $-\mathrm{C}_{20} \mathrm{H}_{34} \mathrm{O}$ - é um diterpeno do tipo labdano, comumente encontrado em diversas famílias de plantas, é o principal diterpeno de várias espécies de sálvia, e está presente em concentrações mais elevadas na Salvia officinalis (Figura 1). 1,3,18,19 É uma espécie da família Lamiaceae (Labiateae), originária do sul da Europa. Apresenta hábito de crescimento herbáceo ou arbustivo de pequeno porte, é planta perene que floresce no Hemisfério Sul entre os meses de agosto e dezembro. ${ }^{20}$

Li et al., ${ }^{21}$ constaram que embora o manool possua atividades desconhecidas do ponto de vista cardiovascular, ele deve ser considerado como fator crucial nos estudos a serem realizados. Além disso, pode ser visto como um novo condutor para o tratamento de doenças cardíacas, merecendo mais investigação. ${ }^{4,21,22} \mathrm{O}$ protocolo experimental incluiu observações sobre os níveis plasmáticos de óxido nítrico (NOx) em animais hipertensos e o impacto da manool na BP de animais após a administração de diferentes doses do composto.

Sabendo que o manool pertence à classe dos compostos diterpênicos, com potencial uso no tratamento da hipertensão, o presente trabalho teve como objetivo avaliar o possível efeito vasodilatador e os mecanismos celulares envolvidos na resposta de relaxamento de anéis aórticos de ratos. Portanto, o objetivo foi avaliar o efeito hipotensor in vivo do manool e verificar o efeito vasorrelaxamento ex vivo em anéis aórticos de ratos.

\section{Materiais e Métodos}

\section{Declaração de Ética e Animais}

As políticas de manejo de animais e procedimentos experimentais foram analisadas e aprovadas pela Comissão de Ética em Experimentação Animal da Faculdade de Medicina de Ribeirão Preto da Universidade de São Paulo (n. 060/210), seguindo as orientações da Diretiva 2010/63 UE da Comissão Europeia. Trinta e quatro ratos Wistar machos (180-220 g) foram acondicionados em condições laboratoriais padrão (ciclo claro/escuro de 12 horas a $21^{\circ} \mathrm{C}$ ) com livre acesso à água e ração. Os animais foram divididos aleatoriamente em cinco grupos de 7 animais para protocolos de pressão 


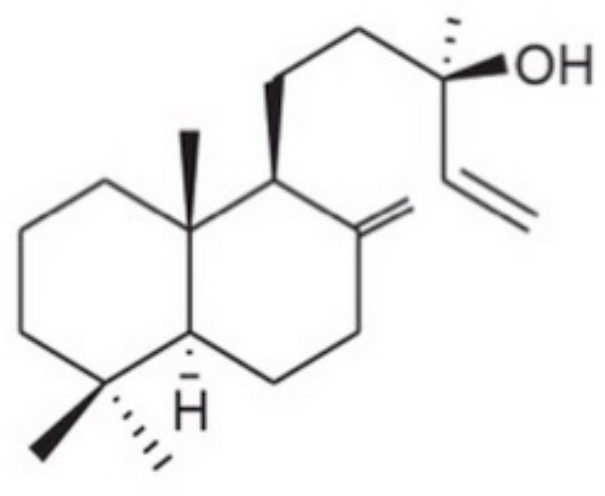

Figura 1 - Estrutura química do Manool. ${ }^{10,11}$

arterial normotensa e hipertensa (veículo normotenso, manool normotenso; veículo hipertenso, manool hipertenso e manool hipertenso + L-NAME). Os animais alocados nos grupos normotensos foram sham-operados, enquanto os animais alocados nos grupos hipertensos foram submetidos ao procedimento cirúrgico 2R1C (dois rins-um-clipe hipertensos) para indução da hipertensão. Utilizou-se um outro grupo de 6 animais que não realizaram nenhum procedimento (intactos) para estudos de reatividade vascular ex vivo.

\section{Fármacos}

Manool, acetilcolina (ACh), 1H-[1,2,4] oxadiazolo[4,3-a] quinoxalina-1-ona (ODQ) e fenilefrina (Phe), da Sigma Chemical Company (St. Louis, MO, EUA); éster metílico de N $\omega$-nitro-L-arginina (L-NAME), obtido na Calbiochem (San Diego, CA, EUA); Vetec Química Fina Ltda forneceu isoflurano da Abbott e todos os sais usados para a preparação da solução de Krebs. Quase todos os fármacos foram preparados com água destilada, sendo o manool solubilizada em dimetilsulfóxido (50 uL) e diluído em etanol/água (2:10, volume total $200 \mathrm{uL}$ ). Para os experimentos de reatividade vascular, $100 \mathrm{uL}$ foram diluídos em 900 uL de água, formando o estoque $\left(10^{-3}\right)$. A partir desse estoque, preparou-se a curva. $\mathrm{O}$ volume usado a partir dessa curva foi de $10 \mathrm{uL}$ em uma cuba de $10 \mathrm{ml}$. Portanto, após tantas diluições, o veículo não promove nenhum efeito na reatividade vascular.

\section{Indução da Hipertensão}

Após anestesia intraperitoneal com cetamina (50 mg/kg) e xilazina $(10 \mathrm{mg} / \mathrm{kg})$, a artéria renal foi exposta. Os grupos hipertensos apresentaram constrição parcial da artéria renal esquerda principal com clipe de prata com abertura de 0,10 mm (2R1C), enquanto os grupos normotensos tiveram a artéria renal esquerda principal isolada, mas não receberam o clipe (sham). Para monitorar o desenvolvimento da hipertensão, a pressão arterial sistólica (PAS) foi medida de forma não invasiva por meio da pletismografia de cauda, uma vez por semana. (Kent Scientific Corporation, Connecticut, EUA). Os ratos 2R1C foram considerados hipertensos com PAS de cauda $\geq$
160 mmHg na 3aㅗ semana após os procedimentos cirúrgicos. Os ratos 2R1C com PAS $<160 \mathrm{mmHg}$ na 3ㄹ semana foram eutanasiados. Menos de $10 \%$ dos animais apresentaram PAS $<160 \mathrm{mmHg}$. Os ratos que foram sham-operados foram incluídos no grupo normotenso.

\section{Efeito do Manool na Pressão Arterial}

Três semanas após a indução da hipertensão, os animais foram anestesiados, e a artéria e veia femoral foram canuladas, respectivamente, para medição contínua da pressão arterial sistólica (PAS) e administração de medicamentos. Após anestesia (uretano, $2 \mathrm{mg} / \mathrm{kg}$, intraperitoneal), canulação vascular e período de estabilização (20 minutos) com registro contínuo da pressão arterial sistólica (PAS) em tempo real, três doses de manool (10, 20 e $40 \mathrm{mg} / \mathrm{kg}$ ) ou veículo (dimetilsulfóxido - DMSO - e água + etanol) foram administrados aos ratos normotensos e hipertensos. Cada dose foi administrada em bolus intravenoso de $200 \mu \mathrm{L}$ e o intervalo entre cada dose consecutiva foi de 6 minutos. Os animais que receberam veículo não receberam manool. Para cada animal, a variação na pressão arterial sistólica $(\triangle \mathrm{PAS})$ foi calculada subtraindo a média dos valores mais baixos de PAS imediatamente após a administração de manool da média dos valores basais de PAS antes do manool ou bolus do veículo. A pressão arterial média foi medida por meio do MP System 100 A (BioPac System, Inc., Santa Bárbara, CA, EUA).

\section{Reatividade Vascular}

Os experimentos foram realizados em anéis aórticos de ratos normotensos. Seis ratos Wistar machos (280-300 g) foram anestesiados com isoflurano inalatório, seguido de exsanguinação da aorta abdominal e toracotomia para retirada da aorta torácica. A aorta torácica foi cuidadosamente dissecada, confirmada como livre de tecido conjuntivo e imediatamente imersa em solução de Krebs. A solução de Krebs era composta por $\mathrm{NaCl}(118,0 \mathrm{mM}), \mathrm{KCl}(4,7 \mathrm{mM})$, $\mathrm{CaCl} 2(2,5 \mathrm{mM}), \mathrm{KH} 2 \mathrm{PO} 4(1,2 \mathrm{mM}), \mathrm{MgSO} 4(1,66 \mathrm{mM})$, glicose $(11,1 \mathrm{mM})$ e NaHCO3 $(25,0 \mathrm{mM})$; a solução tinha $\mathrm{pH}$ 7,4. A aorta torácica imersa em solução de Krebs foi cortada 
em anéis de 4 a $5 \mathrm{~mm}$ de comprimento. Para os testes, o anel com endotélio desnudado foi removido esfregando-se suavemente o vaso da superfície interna com uma haste de aço fina. Esse procedimento remove efetivamente o endotélio, mas não afeta a capacidade do músculo liso vascular de se contrair ou relaxar. Os anéis aórticos foram colocados em 10 $\mathrm{mL}$ de banho orgânico para tecido isolado contendo solução de Krebs, a $37{ }^{\circ} \mathrm{C}$, e $95 \% \mathrm{O}_{2} / 5 \% \mathrm{CO}_{2}(\mathrm{pH} 7,4)$ para medir a força isométrica por meio do equipamento Grass FT03 (Grass Instrument Company, Quincy, MA, EUA). Cada anel foi alongado até o ponto ótimo de estiramento-tensão de 2,0 g, determinado em um estudo piloto, e permaneceram sob esta tensão por $60 \mathrm{~min}$. Durante esse tempo, os tecidos foram lavados a cada 15 minutos. O endotélio foi considerado presente $(E+)$ registrando-se o relaxamento de $80 \%$ induzido por Ach $\left(10^{-6} \mathrm{M}\right)$ após a pré-contração com Phe $\left(10^{-7} \mathrm{M}\right)$. O endotélio foi considerado ausente $(\mathrm{E}-$ ) quando a resposta de relaxamento não ocorreu. Em seguida, cada anel foi lavado e reestabilizado por $30 \mathrm{~min}$. Os anéis aórticos foram contraídos com Phe $\left(10^{-7} \mathrm{M}\right)$ depois que um platô estável foi atingido e as curvas de dose-resposta de manool foram obtidas. Os ensaios de concentração-resposta nos banhos orgânicos foram realizados na presença ou ausência de: L-NAME $\left(2 \times 10^{-4} \mathrm{M}\right)$, um inibidor não específico da óxido nítrico sintase e ODQ $\left(10^{-4} \mathrm{M}\right)$, um inibidor da guanililciclase. ${ }^{20}$ As preparações foram incubadas com os inibidores por $30 \mathrm{~min}$. Não realizamos curvas de dose-resposta com um veículo porque a diluição foi realizada em água. A solução inicial 1 M (50 uL de DMSO + 30 uL de etanol + 120 uL de água) sofreu diluição em série para $10^{-1} \mathrm{M}$ em água.

\section{Medições Plasmáticas Indiretas de NO}

Coletou-se amostras de sangue $(1 \mathrm{ml})$ da artéria femoral após a última curva dose-resposta em veículo normotenso e manool hipertenso, sendo colocadas em tubos heparinizados. Após centrifugação do sangue $\left(3000 \times \mathrm{g}\right.$, 10 minutos, $\left.4{ }^{\circ} \mathrm{C}\right)$, o plasma foi imediatamente imerso em nitrogênio líquido e mantido a $-70{ }^{\circ} \mathrm{C}$ até a dosagem de nitrito e nitrato (NOx). As amostras foram analisadas em duplicata para NOx por ensaio de quimioluminescência à base de ozônio. As amostras plasmáticas foram brevemente tratadas com etanol frio (1 volume de plasma: 2 volumes de etanol por 30 minutos a -20 ${ }^{\circ} \mathrm{C}$ ) e centrifugadas (4000×g, 10 minutos). Os níveis de NOx foram medidos pela injeção de $25 \mu \mathrm{L}$ de sobrenadante em recipiente de purga de vidro contendo $0,8 \%$ de vanádio (III) em $\mathrm{HCl}(1 \mathrm{~N})$ a $90^{\circ} \mathrm{C}$, que reduz o $\mathrm{NOx}$ a óxido nitroso. Uma corrente de nitrogênio foi borbulhada através do recipiente de purga contendo vanádio (III), em seguida através de $\mathrm{NaOH}$ (1 $\mathrm{N}$ ), e então em um analisador de $\mathrm{NO}$ (Sievers ${ }^{\circledR}$ Nitric Oxide Analyzer 280, GE Analytical Instruments, Boulder, CO, EUA).

\section{Análise Estatística}

Os dados são apresentados como média \pm erro-padrão da média. Realizamos análises estatísticas com o teste $\mathrm{T}$ de Student, análise de variância simples (ANOVA), pós-teste de Bonferroni, e ANOVA de duas vias com medidas repetidas, com o pós-teste de Bonferroni para detectar possíveis diferenças entre os valores do estudo. Para cada figura, a legenda descreve qual teste foi realizado para análise.
Considerou-se significativo um p <0,05 (Prism 5.0, GraphPad Software, San Diego, CA, EUA). Um tamanho de amostra de $(\mathrm{N}=5-7)$ por grupo forneceu $95 \%$ de poder com um nível de significância de $0,05 \%$ em protocolos de medição da pressão arterial in vivo. Além disso, um tamanho de amostra de $(\mathrm{N}=$ 6-8) animais por grupo forneceu 95\% de poder com um nível de significância de 0,05 para detectar uma redução relativa de $10 \%$ na contração máxima em vasos pré-contraídos. O número de animais foi escolhido com base na literatura. ${ }^{20,23,24}$

\section{Resultados}

Antes dos procedimentos cirúrgicos, não havia diferenças na PA entre os grupos normotenso e hipertenso. Porém, após

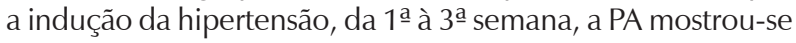
significativamente mais elevada nos ratos hipertensos (130,6 $\mathrm{mmHg}$ versus 193,0 mmHg) (Figura 2).

A avaliação do peso corporal mostrou que, na primeira semana, os grupos apresentavam cargas semelhantes. Porém, ao final de três semanas, o grupo de hipertensos apresentou valores significativamente menores em relação ao grupo de normotensos (Tabela 1).

$\mathrm{Na}$ análise da PAS in vivo, apenas a cirurgia (2R1C) foi capaz de alterar o sangue (veículo normotenso versus veículo hipertenso). O manool promoveu resposta dose-dependente na PAS, reduzindo significativamente a pressão a partir da dose de $20 \mathrm{mg} / \mathrm{kg}$ no grupo normotenso, não havendo diferença entre 20 e $40 \mathrm{mg} / \mathrm{kg}$ neste grupo para o manool. No grupo hipertenso, apenas uma dose menor de manool $(10 \mathrm{mg} / \mathrm{kg})$ reduziu a PAS em comparação ao grupo controle (veículo hipertenso), e a administração prévia de L-NAME preveniu o efeito manool. No grupo hipertenso, o efeito manool não foi dose-dependente (Figura 3).

O NOx plasmático fica um pouco alto no grupo normotenso após a administração de manool, mas não é significativo. Porém, no grupo hipertenso, o manool promoveu aumento nos níveis plasmáticos de NOx (Figura 4).

Sobre os experimentos de reatividade vascular, o manool promoveu um relaxamento dose-dependente em anéis intactos (Figura 5), pré-contraídos com Phe. A incubação com L-NAME ou ODQ bloqueou o relaxamento induzido por manool em anéis com endotélio intacto da mesma forma que a remoção do endotélio (Figuras 6A e 6B).

\section{Discussão}

Pesquisas anteriores mostraram que diterpenos labdanos têm uma ampla gama de efeitos farmacológicos, como a capacidade de inibir a replicação do vírus HIV, prevenir resfriados comuns, ação antimalárica, antibacteriana, antiinflamatória, anti-hiperglicêmica, evitar a disenteria, além de suprimir diversas células cancerosas.6,13 Já em aspecto cardiovascular, evidenciaram: significativa redução de estenose em artérias ateroscleróticas, associada à redução das taxas de reestenose após angioplastia em coelhos; redução da agregação plaquetária in vitro e ação anti-hipertensiva em ratos. ${ }^{13,15-17,25}$ São, portanto, vistos como fonte promissora de novos protótipos para a descoberta e desenvolvimento de novos agentes terapêuticos cardiovasculares. 


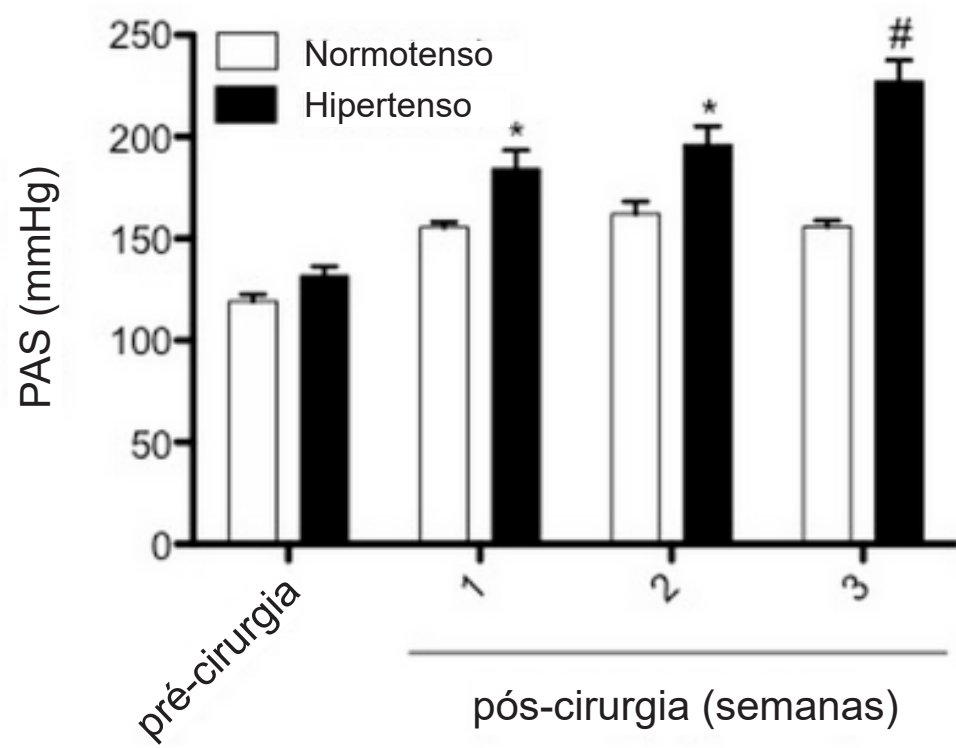

Figura 2 - Evolução temporal da pressão arterial sistólica (PAS) de forma não invasiva em animais normotensos e hipertensos. Os valores representam a média \pm erro padrão da pressão arterial média antes da cirurgia de colocação de clipe (pré-operatório) e três semanas após a cirurgia. * ${ }^{*}<0,05$ e \# $p<0,01$ indicam diferença significativa entre o grupo de hipertensos e normotensos. ANOVA de duas vias, pós-teste de Bonferroni. $n=14$ normotenso e $n=14$ hipertenso.

Tabela 1 - Evolução temporal do peso corporal de animais normotensos e hipertensos

\begin{tabular}{lcc}
\hline \multicolumn{3}{c}{ Evolução do peso corporal $(\mathbf{g})$} \\
\hline Grupos & Inicial & Final \\
\hline Normotenso & $233,4 \pm 7,1$ & $480,2 \pm 10,2$ \\
\hline Hipertenso & $239,4 \pm 7,7$ & $404,8 \pm 18,2{ }^{*}$ \\
\hline $\begin{array}{l}\text { Cada valor representa a média } \pm \text { erro-padrão da média. }{ }^{*} p<0,05 \text { indica } \\
\text { diferença significativa entre o grupo hipertenso e o grupo normotenso. } \\
\text { Teste } t \text { de Student. }\end{array}$
\end{tabular}

Os diterpenos, em particular, estão entre os principais compostos com ligação às propriedades cardiovasculares, como a propriedade vasorrelaxante, inotrópica, diurética e atividade hipotensora. ${ }^{26} \mathrm{~A}$ ação vascular exercida por esses compostos parece envolver múltiplos mecanismos, como endotélio dependente e endotélio independente, aumento de prostaciclinas e bloqueio de canais de cálcio dependentes de voltagem.

No presente estudo, utilizamos o modelo 2R1C para investigar o possível efeito anti-hipertensivo do manool. Esse modelo produziu resultados satisfatórios para a indução de hipertensão, com aumento significativo da pressão arterial em animais, após três semanas da cirurgia. Mesmo na primeira semana após a cirurgia, a PAS de 2R1C foi maior que em um animal normotenso. A PAS encontrada em animais hipertensos está de acordo com outros autores que avaliaram um modelo semelhante..$^{23,27,28}$
Os resultados obtidos após a administração de 3 doses crescentes de manool mostraram que este composto foi capaz de reduzir a PA em ratos normotensos e hipertensos. Em animais normotensos, o manool apresenta efeito doseresposta positivo. Esse achado difere de outros compostos naturais, incluindo o ácido rosmarínico, que reduziu a PA apenas em animais hipertensos. ${ }^{23}$ Esse perfil de resposta não é observado em animais hipertensos, onde o aumento da dose não representa um efeito mais significativo. A $\triangle \mathrm{PAS}$ é a mesma após 10, 20 e 40 mg/kg de manool em animais hipertensos; em outras palavras, independentemente das doses, a pressão arterial máxima era de cerca de 40-50 mmHg. Porém, como no grupo com veículo hipertenso houve redução da PAS, apenas $10 \mathrm{mg} / \mathrm{kg}$ foi capaz de reduzir efetivamente a pressão.

Nossa hipótese para esse efeito anti-hipertensivo da manool baseou-se em estudos recentes sobre a atividade vasodilatadora de diterpenos mediada por NO. ${ }^{13,15,16,26}$ Demonstrou-se que a hipertensão tem forte associação com a formação de espécies reativas de oxigênio (EROs). ${ }^{29}$ Consequentemente, a inativação do NO pelo superóxido induz o desenvolvimento de disfunção endotelial em doenças cardiovasculares. ${ }^{30}$ A propriedade de alguns compostos de aumentar o NO pode ser atraente para reduzir a disfunção endotelial da hipertensão. Nossos achados indicam que o efeito anti-hipertensivo do manool pode ser parcialmente mediado pelo NO, uma vez que a administração de L-NAME antes da injeção de manool bloqueia a redução da PAS em animais hipertensos apenas na dose de $10 \mathrm{mg} /$ kg. Corroborando esses achados, a concentração plasmática de NOx aumentou significativamente apenas nos animais 


\section{Artigo Original}

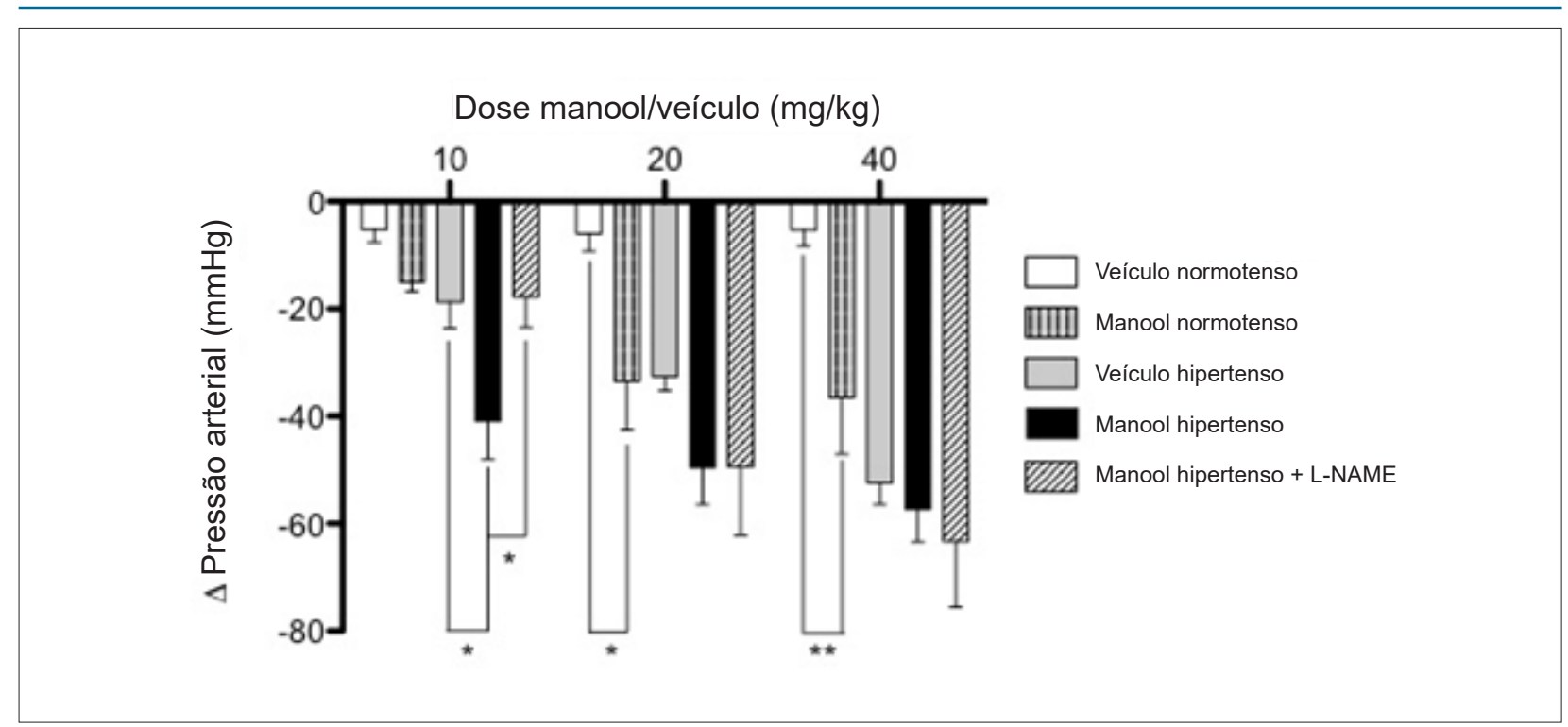

Figura 3 - Alteração da pressão arterial sistólica ( $\triangle P A S)$ após administração de manool ou veículo em ratos normotensos e hipertensos. Os dados são apresentados como média \pm erro-padrão da média. Veículo normotenso $(n=7)$, manool normotenso $(n=7)$, veículo hipertenso $(n=7)$, manool hipertenso $(n=7)$ e manool hipertenso + L-NAME ( $n=7),{ }^{*} p<0,05,{ }^{* *} p<0,01$ indica diferença significativa. ANOVA de duas vias, pós-teste de Bonferroni.

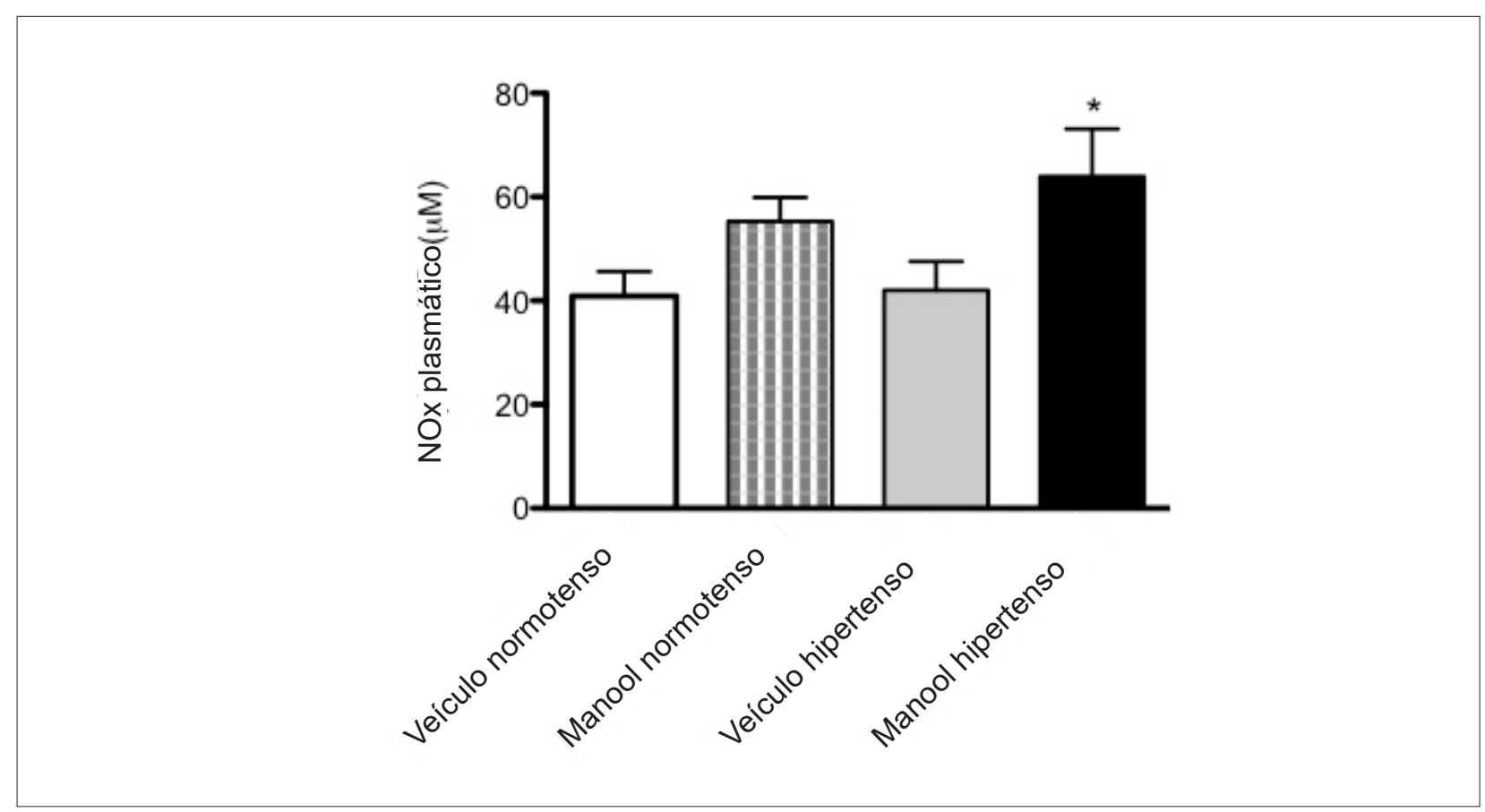

Figura 4 - Niveis plasmáticos de nitrito e nitrato (NOx) em veículos normotensos e manool e veículos hipertensos e animais com manool. ANOVA de uma via, pós-teste de Bonferroni ( $n=7) .{ }^{*} p<0,01$ indica diferença significativa entre veículo hipertenso e manool hipertenso. 


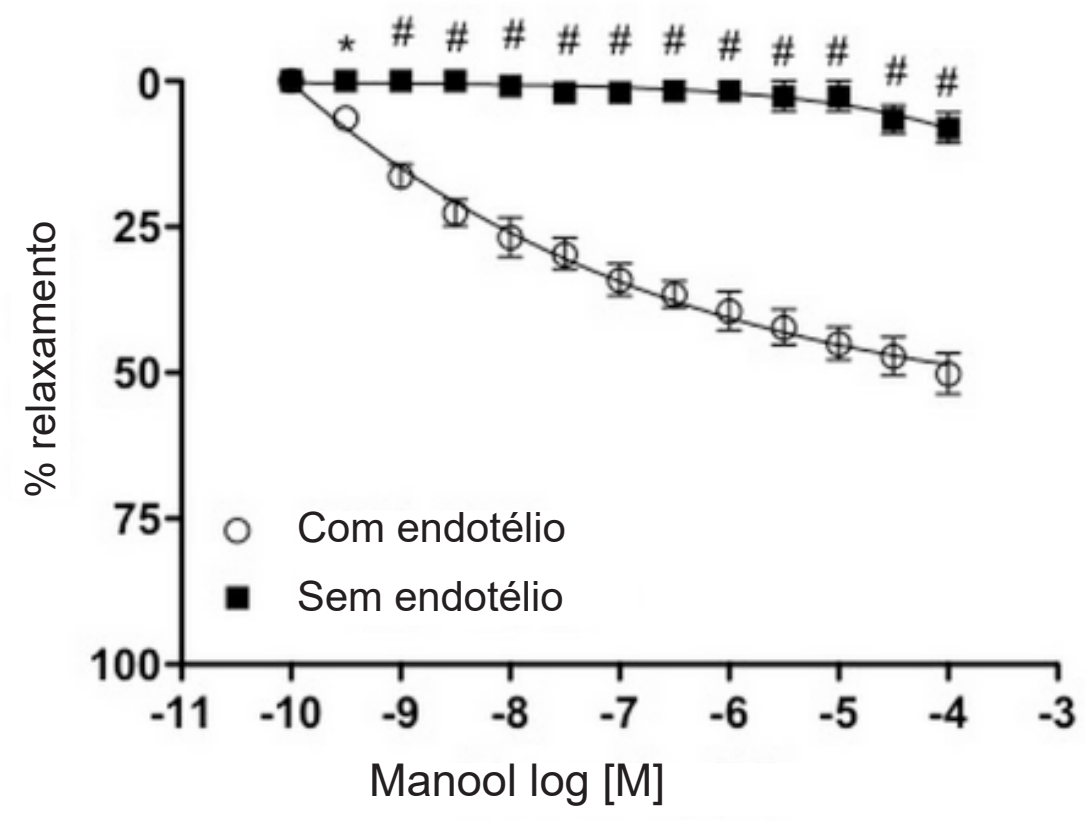

Figura 5 - Curva de relaxamento em anéis de aorta torácica de ratos com endotélio intacto e endotélio desnudado expostos a manool. Os anéis foram pré-contraídos com fenilefrina (Phe) (10-7.M). Todos os valores correspondem à média \pm erro-padrão da média ( $n=6)$. ${ }^{*} p<0,05$ e \# $p<0,001$. ANOVA de duas vias com medidas repetidas e pós-teste de Bonferroni.

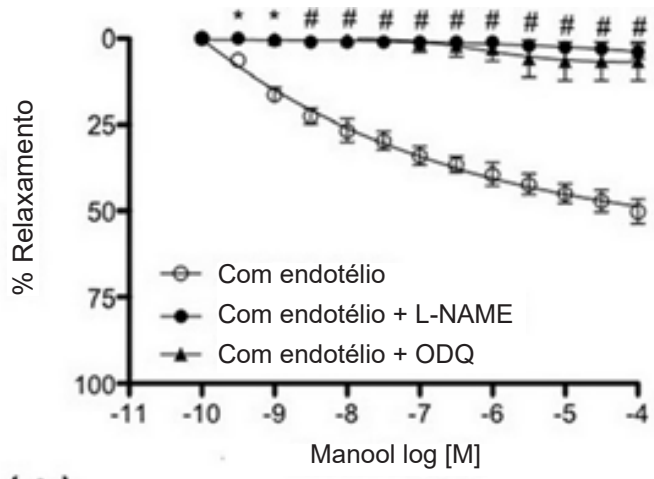

(A)

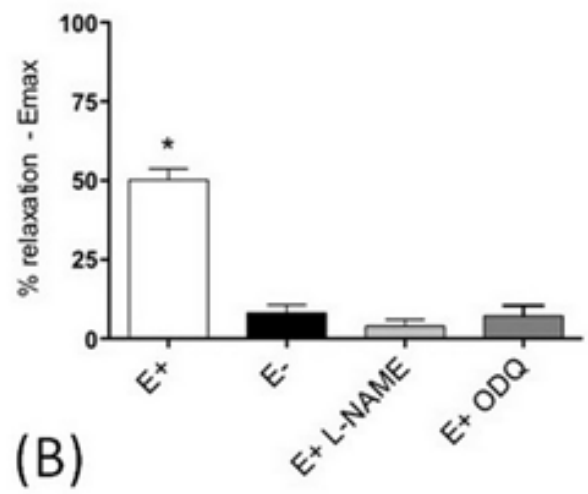

(B)

Figura 6 - Curva de relaxamento em anéis de aorta torácica de ratos com endotélio intacto expostos a manool na presença e ausência de L-NAME (2x10-4 M) ou oxadiazolo[4,3-a]quinoxalina-1-ona (ODQ) (10-4 M). (A) curva dose-resposta e (B) Gráfico de barras Emax. Os anéis foram pré-contraídos com fenilefrina (Phe) (107.M). Todos os valores correspondem à média \pm erro-padrão da média $(n=6)$. * $p<0,05$ e $\# p<0,001$ indicam diferenças significativas entre cada grupo e o grupo controle (vasos com endotélio); ANOVA de duas vias com medidas repetidas e pós-teste de Bonferroni. 
hipertensos que receberam manool. Alguns estudos de NOx no modelo 2R1C mostram que a hipertensão pode reduzir esses níveis, mas nosso achado está em desacordo com esses dados, talvez por causa do tempo da cirurgia 2R1C. ${ }^{31,32}$ Embora o efeito anti-hipertensivo total do manool permaneça desconhecido, outras hipóteses podem ser levantadas, como inibição e modulação da ECA (enzima de conversão da angiotensina). ${ }^{33}$ Demonstrou-se que, no modelo 2R1C, há aumento na atividade da ECA plasmática e alguns peptídeos naturais de arroz, terpenos, fitoestrogênio e compostos polifenólicos podem reduzir a atividade da ECA, ${ }^{20,34,35}$ o que poderia caracterizar esse mecanismo como complementar ao NO na manutenção da PA.

Seria possível atribuir o efeito anti-hipertensivo do manool a um efeito direto na reatividade vascular que não inclui o aumento do NO sistêmico. O presente estudo mostrou que o manool induz o relaxamento aórtico em ratos apenas na presença de endotélio e pré-incubação dos anéis aórticos com inibidores da sintase de óxido nítrico (NOS) ou guanilato ciclase (GC). As propriedades cardiovasculares do diterpeno estão relacionadas ao bloqueio dos canais de $\mathrm{Ca}^{2+}$ e ativação de NO/GMPc (guanosina monofosfato cíclica). ${ }^{13} \mathrm{O}$ endotélio produz vasodilatadores potentes, como o fator relaxante derivado do endotélio (EDRF, NO), prostaciclina e fator de hiperpolarização derivado do endotélio (EDHF). O NO é o mediador predominante na condutância e nas grandes artérias, enquanto o EDHF e a prostaciclina são mais prevalentes nas artérias menores, como os vasos mesentéricos, artérias coronárias e vasos de resistência periférica. ${ }^{36}$ Corroborando nossos achados, alguns têm-se relatos de que alguns diterpenos, como o 14-desoxi-11,12-dihydroandrographolide e 14-desoxyandrographolide dilatam anéis aórticos. O composto 14-desoxi-11,12-dihydroandrographolide teve um efeito hipotensor em ratos anestesiados. Ambos os compostos exercem sua atividade vasorrelaxante pela liberação de $\mathrm{NO}$ e ativação da via da guanilateciclase, bem como pelo bloqueio do influxo de $\mathrm{Ca}^{2+}$ por meio de canais de $\mathrm{Ca}^{2+}$ operados por voltagem e por receptor. ${ }^{13,37-39}$ No presente estudo, também sugerimos que o manool tem um efeito vasorrelaxante dependente do endotélio operando através da via NO/GMPC.

\section{Conclusão}

Em resumo, o manool induz relaxamento vascular dependente do endotélio na aorta de ratos mediado pela via de sinalização NO/GMPc e redução da PA também pelo aumento plasmático de NOx. Esses efeitos em conjunto podem estar envolvidos na modulação da resistência periférica, contribuindo para o efeito anti-hipertensivo desse diterpeno.

\section{Contribuição dos Autores}

Concepção e desenho da pesquisa: Monteiro ASN, Albuquerque AAS, Evora PRB, Ferreira LG, Celotto AC; Obtenção de dados: Monteiro ASN, Campos DR, Albuquerque AAS, Ferreira LG; Análise e interpretação dos dados: Monteiro ASN, Campos DR, Albuquerque AAS, Ferreira LG, Celotto AC; Análise estatística: Monteiro ASN, Campos DR, Albuquerque AAS, Celotto AC; Obtenção de financiamento: Evora PRB, Celotto AC; Redação do manuscrito: Monteiro ASN, Campos DR, Albuquerque AAS, Evora PRB, Ferreira LG, Celotto AC; Revisão crítica do manuscrito quanto ao conteúdo intelectual importante: Albuquerque AAS, Evora PRB, Celotto AC.

\section{Potencial Conflito de Interesses}

Declaro não haver conflito de interesses pertinentes.

\section{Fontes de Financiamento}

O presente estudo foi financiado pelo FAEPA e CNPq.

\section{Vinculação Acadêmica}

Este artigo é parte de dissertação de Mestrado de Ariadne Santana e Neves Monteiro pela Universidade de São Paulo Faculdade de Medicina de Ribeirão Preto.

\section{Referências}

1. Caniard A, Zerbe P, Legrand S, Cohade A, Valot N, Magnard JL, et al. Discovery and functional characterization of two diterpene synthases for sclareol biosynthesis in Salvia sclarea (L.) and their relevance for perfume manufacture. BMC Plant Biol. 2012;12:119.

2. Zerbe P, Hamberger B, Yuen MM, Chiang A, Sandhu HK, Madilao LL, et al. Gene discovery of modular diterpene metabolism in nonmodel systems. Plant Physiol. 2013;162(2):109-91.

3. Saeidnia S, Ghamarinia M, Gohari AR, Shakeri A. Terpenes From the Root of Salvia hypoleuca Benth. Daru. 2012;20(1):66.

4. Wang X, Xu X, Tao W, Li Y, Wang Y, Yang L. A systems biology approach to uncovering pharmacological synergy in herbal medicines with applications to cardiovascular disease. Evid Based Complement Alternat Med. 2012. 2012: p. 519031.

5. Santos MRV, Moreira FV, Fraga BP, Souza DPd, Bonjardim LR, Quintans LJr. Cardiovascular effects of monoterpenes: a review. Rev Bras Farmacognosia. $2011 ; 21: 764-71$
6. Tejera JIJ, Síntesis del diterpeno tipo labdano 12S-Zerumin B. sartenejas:Universidad Simon Bolivar;2005. 103p. 2005, Universidad Simón Bolívar;2005. 103p.

7. Valente ILP, Terpenóides de Euphorbia mellifera Ait.[Tese] Lisboa:Universidade de Lisboa;2007.154p.

8. Viegas Jr C, Bolzani VS, Barreiro EJ. Os produtos naturais e a química medicinal moderna. Química Nova. 2006;29:326-37.

9. Montanari CA and Bolzani VdS. Planejamento racional de fármacos baseado em produtos naturais. Química Nova. 2001;4:105-11.

10. Pinto AC, Silva DHS, Bolzani VS, Lopes NP, Epifanio RA. Produtos naturais: atualidade, desafios e perspectivas. Química Nova. 2002;25:45-61.

11. Funari CS, Ferro VO. Uso ético da biodiversidade brasileira: necessidade e oportunidade. Rev Bras Farmacognosia. 2005;15:178-82.

12. Pletsch M. Compostos naturais biologicamente ativos. A aplicação da biotecnologia à produção de compostos naturais biologicamente ativos. Biotecnologia Ciência \& Desenvolvimento. 1998;1(4):12-5. 
13. Awang K, Abdullah NH, Hadi AH, Fong YS. Cardiovascular activity of labdane diterpenes from Andrographis paniculata in isolated rat hearts. J Biomed Biotechnol. 2012:876458.

14. de Oliveira AP, Furtado FF, da Silva MS, Tavares JF, Mafra RA, Araujo DA, et al. Calcium channel blockade as a target for the cardiovascular effects induced by the 8 (17), 12E, 14-labdatrien-18-oic acid (labdane-302). Vascul Pharmacol. 2006;44(5):338-44.

15. El Bardai S, Morel N, Wibo M, Fabre N, Llabres G, Lyoussi B, et al. The vasorelaxant activity of marrubenol and marrubiin from Marrubium vulgare. Planta Med. 2003;69(1):75-7.

16. Lahlou S, de Barros Correia CA Jr. Santos MV, David JM, David JP et al. Mechanisms underlying the cardiovascular effects of a labdenic diterpene isolated from Moldenhawera nutans in normotensive rats. Vascul Pharmacol. 2007;46(1):60-6.

17. Tirapelli CR, Ambrosio SR, de Oliveira AM, Tostes RC. Hypotensive action of naturally occurring diterpenes: a therapeutic promise for the treatment of hypertension. Fitoterapia. 2010;81(7):609-702.

18. Gong HY, Zeng Y, Chen XY. Diterpene synthases and their responsible cyclic natural products. Nat Prod Bioprospect. 2014;4(2):59-72.

19. Shechter I, West CA. Biosynthesis of Gibberellins. IV. Biosynthesis of cyclic diterpenes from tranx-geranylgeranyl pyrophosphate. JBiol Chem.1969;244(25):3200-9.

20. Campos DR, Celotto AC, Albuquerque AAS, Ferreira LG, Monteiro A, Coelho EB, et al. The Diterpene Sclareol Vascular Effect in Normotensive and Hypertensive Rats. Arq Bras Cardiol. 2017: p. 0.

21. Li X, Xu X, Wang J, Yu H, Wang X, Yang H, et al. A system-level investigation into the mechanisms of Chinese Traditional Medicine: Compound Danshen Formula for cardiovascular disease treatment. PLoS One. 2012. 7(9): p. e43918.

22. Moreira MR, Souza AB, Moreira MA, Bianchi TC, Carneiro LJ, Estrela FT, et al. RP-HPLC analysis of manool-rich Salvia officinalis extract and its antimicrobial activity against bacteria associated with dental caries. Rev Bras Farmacognosia. 2013;23:870-6.

23. Ferreira LG, Evora PRB, Capellini VK, Albuquerque AA, Carvalho MTM, Gomes RAdS, et al. Effect of rosmarinic acid on the arterial blood pressure in normotensive and hypertensive rats: role of ACE. Phytomedicine. 2018;38:158-65.

24. Carvalho MT, Evora PR, Bastos JK, Cunha WR, Andrade E Silva ML, et al. The lignan (-)-cubebin inhibits vascular contraction and induces relaxation via nitric oxide activation in isolated rat aorta. Phytother Res. 2013;27(12):6.

25. Leung PC, Koon CM, Lau CB, Chook P, Cheng WK, Fung KP, etal. Ten years' research on a cardiovascular tonic: a comprehensive approach-from quality control and mechanisms of action to clinical trial. Evid Based Complement Alternat Med. 2013.2013:319703.
26. Mondolis E, Morán-Pinzón JA, Rojas-Marquéz FA, López-Pérez JL, Abad A Amaro-Luis JM, et al. Vasorelaxant effects in aortic rings of eight diterpenoids isolated from three Venezuelan plants. Revista Bras Farmacognosia. 2013;23:769-75.

27. Fazan Jr R, Silva VJD, and Salgado HC. Modelos de hipertensão arterial. RevBras Hipertens.2001;8(1):19-29.

28. Goldblatt H, Lynch J, Hanzal RF, Summerville WW. Studies on Experimental Hypertension: I. The Production of Persistent Elevation of Systolic Blood Pressure by Means of Renal Ischemia. J Exp Med. 1934;59(3):347-9.

29. Paravicini TM, Touyz RM. NADPH oxidases, reactive oxygen species, and hypertension: clinical implications and therapeutic possibilities. Diabetes Care. 2008;31(Suppl 2):S170-80.

30. Shah AM, Channon KM. Free radicals and redox signalling in cardiovascular disease. Heart. 2004;90(5):486-7.

31. Pourshanazari A, Allahtavakoli M, Hassanshahi G. Effects of low-dose morphine on nitric oxide concentration and angiogenesis in two-kidney one clip hypertensive rats. Iran J Bas Med Sci. 2011;14(6):560.

32. Sawant SH, Bodhankar SL. Flax lignan concentrate attenuate hypertension and abnormal left ventricular contractility via modulation of endogenous biomarkers in two-kidney-one-clip (2K1C) hypertensive rats. Ver Bras Farmacognosia. 2006;26(5):601-10

33. Karthik D, Viswanathan $P$, Anuradha CV. Administration of rosmarinic acid reduces cardiopathology and blood pressure through inhibition of p22phox NADPH oxidase in fructose-fed hypertensive rats. J Cardiovasc Pharmacol. 2011;58(5):14-21.

34. Boonla O, Kukongviriyapan U, Pakdeechote P, Kukongviriyapan $\mathrm{V}$ Pannangpetch P, Thawornchinsombut S. Peptides-Derived from Thai Rice Bran Improves Endothelial Function in 2K-1C Renovascular Hypertensive Rats. Nutrients. 2015;7(7):5783-99.

35. Montenegro MF, Pessa LR, Tanus-Santos JE. Isoflavone genistein inhibits the angiotensin-converting enzyme and alters the vascular responses to angiotensin I and bradykinin. Eur J Pharmacol. 2009;607(1-3):173-7 .

36. Shimokawa H, Yasutake H, Fujii K, Owada MK, Nakaike R, Fukumoto Y, et al. The importance of the hyperpolarizing mechanism increases as the vessel size decreases in endothelium-dependent relaxations in rat mesenteric circulation. J Cardiovasc Pharmacol. 1996;28(5):703-11.

37. Zhang C, Kuroyangi M, Tan BK. Cardiovascular activity of 14-deoxy-11,12didehydroandrographolide in the anaesthetised rat and isolated right atria. Pharmacol Res. 1998;38(6):413-7.

38. Zhang CY, Tan BK. Vasorelaxation of rat thoracic aorta caused by 14-deoxyandrographolide. Clin Exp Pharmacol Physiol. 1998;25(6):424-9.

39. Zhang CY, Tan BK. Effects of 14-deoxyandrographolide and 14-deoxy11,12-didehydroandrographolide on nitric oxide production in cultured human endothelial cells. Phytother Res. 1999;13(2):157-9. 